\title{
O CONSUMO DE BEBIDA ALCÓOLICA PELAS GESTANTES: UM ESTUDO EXPLORATÓRIO
}

\author{
The Alcoholic Beverage Consumption by the Pregnant Women: \\ an Exploratory Study \\ El Consumo de Bebida Alcohólica por Gestantes: \\ un Estudio Exploratório
}

Thalita Rocha Oliveira

Sonia Mara Faria Simões ${ }^{2}$

\begin{abstract}
Resumo
A pesquisa de natureza quantitativa de tipo exploratório teve como objetivo geral discutir os motivos/fatores que levam as gestantes a consumirem bebidas alcoólicas. 0 cenário foi o ambulatório de pré-natal de um hospital universitário do Estado do Rio de Janeiro. Fizeram parte do estudo 40 gestantes, tendo como instrumento de coleta de dados dois formulários com perguntas fechadas. A análise dos dados evidenciou que $10 \%$ das gestantes possuíam o hábito de consumir bebidas alcoólicas moderadamente, sendo o principal fator motivacional a presença em festas e comemorações, além de se sentirem felizes e descontraídas no momento do consumo. Quanto ao conhecimento sobre a teratogenia do álcool, constatou-se que apenas metade das gestantes que consumiram bebidas alcoólicas acreditava que esta prática poderia afetar seu filho. 0 estudo revelou que dados como o estilo de vida devem ser valorizados em assistência pré-natal pela enfermeira, direcionando ações educativas que visem à qualidade de vida do núcleo familiar.
\end{abstract}

Palavras-chave: Alcoolismo. Gravidez. Cuidado Pré-natal. Síndrome Alcoólica Fetal e Enfermagem.

\begin{abstract}
The research of quantitative and explorer nature had the general objective to argue the reasons/factors that take the pregnant women to consume alcoholic beverage. The scene was the Prenatal clinic of the Hospital Antonio Pedro, Niterói. Forty future mothers had been part of the study, having as instrument of collection of data two forms with closed questions. The analysis of the data evidenced that $10 \%$ of the pregnant women had the habit to consume moderately alcoholic beverage, being the main reason the presence in parties and commemorations beyond felt happy and relaxed at the moment of consumption. About the knowledge on the teratogen of the alcohol, it was evidenced that only half of the pregnant women that had consumed alcoholic beverage believed that this practical could affect its son. The study disclosed that data as the life style must be valued in Prenatal assistance being able to direct educative actions, aiming at the quality of life of the familiar nucleus.
\end{abstract}

Keywords: Alcoholism. Pregnancy. Care Prenatal. Fetal alcoholic Syndrome. Nursing.

\section{Resumen}

La investigación es de naturaleza cuantitativa del tipo exploratória tuvo como objetivo general discutir las razones/los factores que llevan las gestantes a consumir bebida alcohólica. El escenario fue la clínica prenatal de un Hospital Universitario del Estado del Rio de Janeiro - Brasil. Participaran del estudio 40 gestantes, teniendo como instrumento de colecta de datos dos formularios con preguntas cerradas. El análisis de los datos evidenció que $10 \%$ de las gestantes possuíam el hábito de consumir la bebida alcohólica de forma moderada, siendo el factor principal motivacional la presencia en fiestas y conmemoraciones además las gestantes se sienten felizesy relajadas, en el momento del consumo. Cuánto al conocimiento en la teratogênia del alcohol, se evidenció que solamente la mitad de las gestantes que habían consumido bebida alcohólica creyó que esta práctica podría afectar a su niño. El estudio reveló que datos como el estilo de vida debe ser valorado en asistencia prenatal por la enfermera, direccionando las acciones educativas teniendo como objetivo la calidad de vida del núcleo familiar.

Palabras clave: Alcoholismo. Gestación. Cuidado Prenatal. Síndrome Alcohólico Fetal. Enfermería. 


\section{INTRODUÇÃO}

Ao longo da história, a humanidade sempre recorreu ao uso de substâncias psicoativas com as mais diversas finalidades. Estas substâncias são utilizadas isoladamente ou, com maior freqüência, associadas a outros meios de alteração da consciência/ ânimo, tais como: música, dança, meditações, provocação de dor, entre outros. Com algumas exceções, o uso tem sido regulado por normas sociais ou conjunto de práticas onde se manifestam os valores intrínsecos à cultura, entre eles a manutenção da coesão social e o bem-estar físico e psíquico de seus integrantes ${ }^{1}$.

Atualmente, o Brasil aumentou a sua produção de bebida alcoólica devido ao aumento do consumo que dobrou nos últimos cinco anos, sendo as mulheres e os jovens os maiores contribuintes neste processo. Associado a este aumento na produção mundial de bebidas alcoólicas, observa-se, segundo estatísticas, um elevado surgimento de problemas relacionados ao acréscimo do consumo. Os gastos referentes à gama de conseqüências e prejuízos decorrentes do alcoolismo alcançam hoje no Brasil o equivalente a $7 \%$ do PIB, ou seja, cerca de 100 bilhões de reais². No período de 1988 a 1999, o álcool foi responsável por cerca de $90 \%$ de todas as internações hospitalares em virtude das dependências, perfazendo um total de 62.242 internações em comparação a 3.062 no que se refere a outros diagnósticos de internações por substâncias psicoativas ${ }^{3}$.

0 consumo de álcool é um hábito constante na vida de mulheres em idade reprodutiva, isto porque na sociedade moderna as mulheres ocupam de forma progressiva o mercado de trabalho, o que modifica, conseqüentemente, o seu papel social.

Assim, nas sociedades modernas, os desenvolvimentos científicos, culturais e sócio-econômicos transformaram os estereótipos tradicionais femininos, resultando em um efeito indireto sobre o consumo de produtos que acarretam dependência, como o tabaco e o álcool, explicando de certa forma o aumento de toxicomanias na população feminina ${ }^{4}$.

A maioria das mulheres diminui a quantidade de bebida alcoólica durante a gestação, principalmente ao saber que está grávida. Em estudos, constatou-se que $44 \%$ bebiam pelo menos 1 drink por dia antes da gestação, sendo este índice reduzido para $37 \%$ durante o primeiro mês, $21 \%$ durante o segundo mês e $14 \%$ ao final do terceiro mês de gestação. As mulheres que mantinham o consumo de bebidas, uma média de 1 drinkpor dia, até o final do terceiro trimestre representavam $5 \%{ }^{5}$.

Esses índices são significativos na medida em que o primeiro trimestre de gestação se caracteriza pela formação das estruturas do feto, como, por exemplo, o desenvolvimento do tubo neural, em que todo este processo pode ser afetado.

Além disso, o uso abusivo do álcool nas primeiras semanas de gestação pode estar relacionado com os casos de abortamento espontâneo, e seu consumo entre a $3^{\circ}$ e $8^{\circ}$ semana pode causar maior risco de deformações físicas. De acordo com pesquisas realizadas em 24.679 gestantes que realizaram o pré-natal no Aarhus University em Denmark entre os anos de 1989 e 1996, concluiu-se que mulheres que consomem uma quantidade maior ou igual a 5 drinks por semana apresentam maiores riscos de aborto espontâneo no $1^{\circ}$ trimestre de gestação $0^{6}$.
Já é constatado que o efeito do álcool no recém-nascido é manifestado através da Síndrome Fetal Alcoólica, que afeta $33 \%$ das crianças nascidas de mães que fizeram uso de mais de $150 \mathrm{~g}$ de etanol por dia. Esta síndrome é caracterizada por: retardo no crescimento intra-uterino, alterações na coordenação motora, anomalias articulares, malformações cardíacas, redução da capacidade intelectual, entre outros. Além disso, filhos de mulheres que consumiram moderadamente bebida alcoólica podem apresentar agitação, deficiência de sucção durante 0 aleitamento, irritabilidade, sudorese e padrões anormais de sono, caracterizando um quadro de síndrome da abstinência ${ }^{7}$.

Diante do exposto, surgiram as seguintes hipóteses: as gestantes possuem conhecimento sobre a teratogenia do álcool; e existem motivos/fatores que levam as gestantes a consumirem bebidas alcoólicas. Desta forma, este estudo teve por objetivos identificar as gestantes que consomem bebidas alcoólicas durante a gestação; caracterizar o grau de conhecimento da gestante sobre as conseqüências da ação do álcool no feto e no seu futuro desenvolvimento; e conhecer os motivos/fatores relacionados com o consumo de bebida alcoólica na gestação.

A presente pesquisa se faz relevante na medida em que 0 Sistema de Risco Comportamental informa que, entre os anos de 1985 e 1988, verificou-se que 25\% das 1.712 gestantes relataram terem ingerido bebida alcoólica ${ }^{7}$. Além disso, durante 0 ano de 2001, identificaram-se 22,2\% de gestantes, de um total de 450 sujeitos, que consumiram bebidas alcoólicas em nível considerado prejudicial para o feto, atendidas em uma maternidade do estado de São Paulo ${ }^{8}$.

Portanto, a prevenção é a melhor solução para reduzir riscos. Na França, em 1988, ocorreu uma diminuição da quantidade de consumo de bebida alcoólica pelas mulheres em virtude da mudança de comportamento conseqüente à sensibilização durante o acompanhamento no pré-natal ${ }^{9}$. Infelizmente durante a prática acadêmica e profissional não foi observada com freqüência a atuação do profissional de saúde, enfatizando a questão dos danos causados pelo uso de drogas lícitas na gestação.

Além disso, ao buscar conhecer os motivos/fatores que levam as gestantes a consumirem bebida alcoólica, mesmo tendo informações sobre o risco que esta prática proporciona, pode se ter uma melhor orientação na formulação de políticas públicas comprometidas com a promoção, prevenção e tratamento deste "vício", conforme preconiza Ministério da Saúde (2005).

\section{METODOLOGIA}

Este estudo baseia-se em uma abordagem quantitativa do tipo exploratório. 0 cenário da pesquisa foi o ambulatório de pré-natal de um hospital universitário no Estado do Rio de Janeiro. Participaram do estudo 40 gestantes que realizaram o acompanhamento de pré-natal nesta instituição, havendo como critérios de exclusão as gestantes menores de 18 anos, analfabetas e as que não possuíam condições psíquicas de fornecer informações ao pesquisador.

A entrada no campo da pesquisa se deu a partir da aprovação do protocolo de pesquisa encaminhado ao Comitê de Ética em Pesquisa da Instituição a fim de cumprir o que preceitua a Resolução no 196/96 do Conselho Nacional de Saúde, que 
regulamenta a pesquisa envolvendo seres humanos. A autorização dos sujeitos do estudo para participação foi registrado através do termo de consentimento livre e esclarecido, o qual foi apresentado às gestantes previamente à entrevista, bem como os objetivos do estudo e a certificação de anonimato das informações.

0 período de coleta de dados teve início em abril de 2006 e término em junho de 2006, perfazendo um total de 10 dias. Esta etapa foi implementada da seguinte forma: primeiramente, aplicou-se um formulário contendo informações de identificação da gestante, dados sócio-demográficos, dados obstétricos e informações referentes aos hábitos de tabagismo e consumo de bebidas alcoólicas pelos familiares e pelas gestantes.

Em seguida, foi aplicado o teste americano T-ACE (Tolerance, Annoyed, Cut down e Eyeopener) (ANEX0 1) ${ }^{10}$. Este teste foi extraído de uma dissertação de mestrado ${ }^{8}$ no qual o pesquisador procedeu à validação do mesmo para uso no Brasil pelo cálculo estatístico de sensibilidade, especificidade, valor preditivo positivo e valor preditivo negativo. Ele é composto por quatro perguntas principais, as quais buscam informações sobre a tolerância da bebida alcoólica, a existência de conflitos com familiares devidos ao consumo excessivo, a sua percepção sobre a ingestão em demasia do álcool e, por fim, a existência da necessidade de continuar a beber durante o dia. A distribuição dos pontos para as questões ocorre da seguinte maneira: a primeira questão sobre tolerância vale 2 pontos; se a gestante responder que consome mais de duas doses diárias ( $28 \mathrm{~g}$ de álcool absoluto) de bebida alcoólica, a questão terá a pontuação máxima, ou seja, 2 pontos. Da segunda à quarta pergunta, a pontuação é de 1 ponto, ou seja, a cada resposta positiva será somado 1 ponto. Ao final das quatro perguntas pode-se obter uma pontuação total para o questionário que pode variar de 0-5 pontos. A gestante que obtiver um escore maior ou igual a 2 pontos é considerada como um caso positivo para o teste T-ACE.

Vale lembrar que se considera a existência, em média, de 14 gramas de álcool absoluto em um drink, o que é equivalente a 360 $\mathrm{ml}$ de cerveja, $120 \mathrm{ml}$ de vinho ou $36 \mathrm{ml}$ de licor e similares ${ }^{8}$.

Após a realização do teste, ocorrendo a identificação de casos positivos, seria então realizada a aplicação de um segundo formulário buscando "identificar os possíveis fatores relacionados ao consumo de bebida alcoólica na gestação e o conhecimento das gestantes sobre os malefícios do consumo de álcool para o feto e para o desenvolvimento futuro da criança". Porém, na aplicação do teste T-ACE nas 40 gestantes, não houve a identificação de casos positivos. Optou-se então por aplicar o segundo formulário em gestantes que fizeram consumo de bebidas alcoólicas ocasionalmente, ou seja, consumo em festas e comemorações, ou freqüentemente, caracterizado como consumo de bebidas alcoólicas aos finais de semana.

Portanto, à princípio, a pesquisa tinha como objetivo aplicar o teste T-ACE em uma amostra com valor científico com o fim de identificar o consumo alcoólico de risco (casos positivos) que pode ocasionar o desenvolvimento da Síndrome Alcoólica Fetal. Tendo em vista o tempo acadêmico do término da pesquisa, aproveitaram-se as clientes identificadas através do formulário 1 com consumo moderado de bebida alcoólica para realizar o estudo exploratório acerca dos motivos/fatores relacionados ao consumo de bebida alcoólica no período gestacional e do conhecimento sobre as conseqüências deste hábito.

Além da aplicação destes instrumentos, a pesquisadora buscou informações em prontuário médico do registro de profissionais de saúde em relação aos hábitos de vida das gestantes na ficha de prénatal, cujo preenchimento é realizado na primeira consulta.

Os dados foram apresentados em freqüência absoluta e relativa, sendo organizados como temáticas que emergiram de questões dos formulários. A discussão se deu à luz do referencial teórico da temática em que foi utilizada como banco de dados a rede Scielo.

Para o cálculo do tamanho da amostra consideraram-se em torno de $30 \%$ das gestantes que realizaram acompanhamento pré-natal num período de 10 dias. Totalizou-se uma população estimada de 250 atendimentos efetuados no mês anterior ao início da pesquisa de acordo com dados fornecidos pelo Controle de Faturamento Ambulatorial do hospital de estudo.

\section{APRESENTAÇÃO E DISCUSSÃO DOS RESULTADOS}

Das 40 gestantes que participaram da pesquisa, foram identificados quatro sujeitos que possuíam o hábito de consumir bebidas alcoólicas, porém em níveis não caracterizados como de dependência.

Nos dados sócio-demográficos representados pela Tabela 1, $61,1 \%$ das gestantes entrevistadas que não fizeram uso de bebida alcoólica durante a gravidez encontram-se na faixa etária de 1829 anos, enquanto, das que fizeram consumo ocasional ou freqüente, metade dos sujeitos (50\%) está na faixa etária de 18-29, e a outra metade $(50 \%)$ de 30-39 anos.

Assim, as mulheres adolescentes parecem iniciar e abusar da ingestão de álcool em virtude de uma combinação de aspectos de vulnerabilidade individual e da exposição destes por intermédio de grupos de amigos ${ }^{11}$.

Com relação ao estado civil em ambas as categorias, consumidoras ou não, identificou-se que a maioria, $80,5 \%$ e $75 \%$ respectivamente, estava casada.

No que diz respeito à escolaridade, a maior parte das gestantes que não fizeram ingestão de bebida durante o período gestacional concluiu o ensino médio, representando 36,1\%. Já as gestantes que possuíam o hábito de beber na gestação, todas concluíram apenas o ensino fundamental.

Quanto à renda familiar das entrevistadas, 55,5\% das grávidas que não consumiram bebidas possuem renda de 3-6 salários mínimos, enquanto $75 \%$ das gestantes consumidoras possuem baixa renda, correspondendo a 1-2 salários mínimos.

É interessante ressaltar que estudos ${ }^{12}$ apontam para uma maior ocorrência de consumo de bebidas alcoólicas na gestação em mulheres solteiras, quando comparadas com as casadas, na medida em que a gestação destas está associada, freqüentemente, a outros fatores de risco para a ingestão de bebidas alcoólicas, como a baixa escolaridade, baixo nível sócioeconômico e gravidez indesejada. Acrescentando 11:72, "Fatores de ordem sócio-cultural exercem poderosa influência sobre o padrão e o grau de ingestão de álcool pelas mulheres, alterando a vulnerabilidade ao desenvolvimento de problemas decorrentes 
Tabela 1:

Condições sociais das gestantes segundo o consumo de bebidas alcoólicas durante a gestação, no APN HUAP/UFF*, abr-jun 2006.

\begin{tabular}{ccccc}
\hline & Sem Consumo** & \multicolumn{2}{c}{ Com Consumo*** } \\
$\mathrm{N}$ & $\%$ & $\mathrm{~N}$ & $\%$ \\
\hline
\end{tabular}

\section{Faixa Etária}

$18-29$

30-39

$40 \mathrm{a}+$

13

61,1
36,1
2,8

250,0

Estado Civil

Casada

Solteira

Biótipo

Branca

Negra

Parda

7

19,5

$\begin{array}{ll}3 & 75,0 \\ 1 & 25,0\end{array}$

\section{Escolaridade}

Fundamental Incompleto

Fundamental Completo

Médio Incompleto

Médio Completo

Superior Incompleto

$\begin{array}{cccc}6 & 16,6 & - & - \\ 15 & 41,7 & 2 & 50,0 \\ 15 & 41,7 & 2 & 50,0\end{array}$

Renda Familiar

1-2 salários mínimos

3-6 salários mínimos

13

\section{6,1}

$$
3
$$

75,0

Acima de 7 salários mínimos

20

55,5

1

25,0

Não soube informar

2

5,5

\section{Ocupação}

Com vínculo empregatício

Sem vínculo empregatício

Estudante

1

2,8

Reside com

Um familiar

Dois ou mais familiares

$\begin{array}{ll}17 & 47,4 \\ 13 & 36,0 \\ 6 & 16,6\end{array}$

$\begin{array}{ll}4 & 100,0\end{array}$

\begin{tabular}{lcccc} 
Reside com & 7 & 19,5 & 1 & 25 \\
Um familiar & 29 & 80,5 & 3 & 75 \\
Dois ou mais familiares & 29 \\
\hline
\end{tabular}

*sigla utilizada para denominar o local da pesquisa, Ambulatório de Pré-Natal do Hospital Universitário Antônio Pedro da Universidade Federal Fluminense. ** gestantes que não realizaram, em nenhum momento da gestação, consumo de bebidas alcoólicas. *** gestantes que consumiram álcool ocasionalmente ou freqüentemente durante a gestação.

do consumo". O fator sócio-cultural está intimamente relacionado ao nível de formação educacional da população.

Nesta pesquisa, os dados apontaram que houve uma igualdade numérica entre as mulheres que consumiram bebidas alcoólicas no período gestacional no que se refere à situação marital, porém foi também observado que as gestantes consumidoras de bebidas haviam completado apenas 0 ensino fundamental $\mathrm{e}$ possuíam baixa renda, corroborando os autores acima.

Da amostra estudada, foi identificado que as gestantes que consumiram bebidas alcoólicas durante a gestação convivem com esposo ou pai que possuem o mesmo hábito. Este fato se confirma em pesquisa realizada ${ }^{11}$ na qual se constatou que existe uma forte influência da família no primeiro contato de algumas mulheres com o álcool, não apenas na oferta do álcool, mas ainda na demonstração de atitudes permissivas, revelando a falta de esclarecimento a respeito do consumo de álcool e de suas conseqüências. Além disso ${ }^{4}$, ocorre uma considerável influência de parentes na adoção de determinados comportamentos diante da bebida alcoólica. $\mathrm{Na}$ realidade, as mulheres que consomem bebidas alcoólicas em demasia encontram-se, significativamente, em maior número em famílias onde existem hábitos de bebedeiras acentuados.

No estudo, evidenciou-se que das entrevistadas que costumavam ingerir bebidas alcoólicas na gestação, três realizaram consumo ocasional e uma possuía este hábito freqüentemente. 0 consumo ocasional significa o realizado em festas e comemorações, e o freqüente, o consumo feito aos finais de semana.

Mesmo não ocorrendo a identificação de nenhuma gestante positiva para o teste americano T-ACE, ou seja, aquelas que 
realizam consumo de $28 \mathrm{~g}$ álcool/dia proporcionando o risco de desenvolvimento de Síndrome Alcoólica Fetal, surgiram gestantes que consomem moderadamente bebidas alcoólicas durante o período gestacional. 0 pequeno consumo de álcool parece não estar associado a alterações de crescimento e desenvolvimento, porém o seu efeito teratogênico não pode ser descartado, havendo aumento da incidência de malformações fetais em bebedoras consideradas moderadas ${ }^{13}$. Acrescentando, o uso moderado pode estar relacionado a abortos espontâneos ${ }^{14}$.

Os efeitos do etanol sobre o feto dependem da dose diária ingerida, efeitos esses evidentes quando o consumo ocorre no primeiro trimestre de gestação ${ }^{15}$.

É relevante salientar que não existe um consenso sobre a quantidade limite de álcool que proporcione alterações morfológicas e funcionais, sendo dependente de fatores individuais de cada mulher e da idade gestacional. Portanto, mesmo quantidades pequenas em algumas gestantes podem ocasionar danos fetais.

Das 36 gestantes que responderam não ingerirem bebidas alcoólicas na gestação, 10 o fizeram somente até a confirmação da gravidez. Todas responderam que a interrupção ocorreu por vontade própria, pois sabiam que esta prática, assim como o tabagismo, podem prejudicar o desenvolvimento do bebê. Ou seja, os dados acima demonstram que as gestantes, apesar de não possuírem conhecimentos técnico-científicos sobre a teratogenia do álcool, sabem que se deve realizar a abstinência no período gestacional devido aos seus malefícios. As outras 26 gestantes que não possuíam o hábito de beber apresentavam alguma patologia que as levaram a parar de beber ou por não apreciarem as bebidas alcoólicas.

Ao se verificar o registro de profissionais de saúde quanto aos hábitos de vida das gestantes no tocante ao consumo de bebidas alcoólicas, observou-se que na maioria dos prontuários não havia informações. Isto pode indicar que o consumo de bebidas alcoólicas pelas gestantes está sendo subdiagnosticado pelo serviço de pré-natal, isto devido ao despreparo de profissionais em realizar uma investigação mais aprimorada. Este fato se confirma na medida em que ${ }^{16}$ ocorre, em geral, falta de treinamento dos profissionais da área de saúde para formas mais eficazes de detecção do consumo de bebidas alcoólicas pelas mulheres. Ao mesmo tempo, o consumo pode ser detectado, mas não registrado nos prontuários, significando uma desvalorização dos dados referentes ao estilo de vida das mulheres como fator influente no desenvolvimento gestacional.

Os dados a seguir dizem respeito aos aspectos psicológicos e aos motivos/fatores relacionados pelas gestantes quanto ao consumo de bebidas alcoólicas na gestação, e ao seu conhecimento sobre a teratogenia do álcool.

A maioria das gestantes que possuía o hábito de consumir bebidas alcoólicas caracterizava o estado emocional em facilidade de se estressar com pequenas coisas como, por exemplo, desentendimentos com familiares por simples motivos.

Cabe ressaltar que as consultas de pré-natal têm uma nova tendência a aproveitar o período gestacional para proporcionar às mulheres, principalmente as de baixa condição sócioeconômica, um atendimento ampliado, denominado Assistência Integral à Mulher ${ }^{17}$. Logo, deve-se não só estar atento quanto às questões ginecológicas e obstétricas, mas também aos aspectos psicológicos ajudando e orientando na resolução de conflitos e problemas, que podem em graus variados influenciar na evolução gestacional.

Evidenciou-se que todas as gestantes que possuíam 0 hábito de consumir bebidas alcoólicas na gestação o fazem na presença de amigos, família e companheiros. Isto corresponde ao caráter recreacional associado às bebidas alcoólicas, sendo o consumo moderado pelas gestantes aceito de alguma forma pela sociedade.

As drogas ilícitas e lícitas como as bebidas alcoólicas e o tabaco são onipresentes nas sociedades modernas ocidentais. 0 álcool é socialmente aceito para celebrar aniversários, para regar um banquete ou um almoço familiar ou para brindar entre amigos ${ }^{4}$.

No que se refere aos sentimentos vivenciados pelas entrevistadas no momento do consumo de bebidas alcoólicas, percebe-se que a maioria das gestantes encontra-se feliz e descontraída no momento em que está consumindo bebida junto de seus amigos e familiares. Estes sentimentos confirmam que as gestantes não apresentam um perfil de consumo crônico de bebidas alcoólicas. Isto porque, segundo estudos de comorbidade, "a usuária de álcool tem a depressão como diagnóstico psiquiátrico associado mais freqüentemente"11:77.

A presença em festas e comemorações foi o motivo/fator encontrado pelas gestantes que as fizeram consumir bebidas alcoólicas na gestação. Esta prática é um fato predominantemente social em que as ocasiões de ingestão de bebidas alcoólicas funcionam como expressão e apoio à estrutura social existente, estimulando interações sociais e fortalecendo identificações e solidariedade coletiva ${ }^{18}$.

Em relação ao conhecimento sobre os efeitos da bebida para o feto na gestação, metade das gestantes que possuem 0 hábito de consumir bebidas alcoólicas mesmo durante a gestação acredita que esta prática pode afetar seu filho de alguma maneira; e a outra metade diz não acreditar que o álcool pode ocasionar algum malefício durante o período gestacional ao feto.

Isto reflete que as informações sobre os malefícios das bebidas alcoólicas e do tabaco usado durante a gestação não estão sendo vinculados de forma adequada, tanto pelos meios de comunicação quanto por parte dos profissionais de saúde ao realizar educação em saúde nas consultas de pré-natal sobre as conseqüências do uso de tais substâncias. Além disso, pode-se ter como outro fator explicativo as diferenças no grau de escolaridade e no ambiente social em que estas gestantes se encontram inseridas, proporcionando conhecimento diferenciado.

A maioria das gestantes acha que o consumo de bebida alcoólica pode ser responsável pela malformação física do feto. Pode-se deduzir que para o censo comum qualquer uso de substâncias durante o período gestacional, farmacológico ou não, causa malformações físicas como o exemplo dos efeitos do uso da medicação talidomida que ficou popularmente conhecida.

Por fim, foi analisado o conhecimento das gestantes sobre que efeitos a bebida alcoólica acarreta no desenvolvimento infantil. As mulheres referiram a dificuldade de concentração e o retardo mental como possíveis efeitos proporcionados pelo consumo de álcool na gestação ao desenvolvimento infantil da criança. "Aos 4 anos de idade, as crianças que 
foram expostas moderadamente ao álcool no período prénatal apresentavam-se menos atentas e mais ativas durante observações de perto em casa [...]" 5:1311.

É interessante ressaltar que uma gestante referiu não possuir nenhum conhecimento das conseqüências da ingestão de bebidas alcoólicas para a vida futura da criança, demonstrando que é necessário, nas consultas de acompanhamento pré-natal, não só interrogar as mulheres quanto ao uso e abuso de bebidas alcoólicas e do cigarro, mas também orientá-las das conseqüências no período gestacional.

\section{CONSIDERAÇÕES FINAIS}

0 uso de substâncias psicoativas é regulado por normas sociais ou conjunto de práticas no qual se manifestam os valores intrínsecos à cultura, entre eles a manutenção da coesão social e o bem-estar físico e psíquico de seus integrantes. Com a evolução da sociedade moderna e a inclusão da mulher no mercado de trabalho, que se intensificou a partir da segunda metade do século passado, observou-se o aumento do uso de drogas lícitas como o álcool e o tabaco pelas mulheres o que representava uma prática caracteristicamente masculina. Logo este hábito passou a ser uma constante na vida de mulheres em idade reprodutiva, englobando também as gestantes.

De acordo com a literatura estudada, preconiza-se a abstinência total de substâncias lícitas, na medida em que não existe um valor seguro da quantidade que poderia ser consumida sem provocar danos ao desenvolvimento fetal ou mesmo intercorrências obstétricas, pois existem diferenças orgânicas que variam de indivíduo para indivíduo. Ao conhecer o que influencia as gestantes a ingerirem bebidas, pode-se planejar e desenvolver ações que facilitem a abordagem destas mulheres quanto às orientações necessárias durante o pré-natal.

A metodologia possibilitou o levantamento de dados significativos. Da amostra estudada, detectou-se que 10\% realizaram consumo de bebidas na gestação, sendo o principal fator contribuinte para tal prática a presença em festas e comemorações na companhia de familiares e amigos, justificando-se o caráter social e recreacional das bebidas

\section{Referências}

1.Ministério da Saúde (BR). Não existe sociedade sem droga. [on-line] 2005. [citado 28 maio 2005] Disponivel em: http//:www.saude.gov.br.

2. Lima JMB. Alcoologia: uma visão sitêmica dos problemas relacionados ao uso e abuso do álcool. Rio de Janeiro (RJ): UFRJ/EEAN; 2003.

3. Noto AR, Moura YG, Nappo S, Gualduroz JCF, Carlini EA. Internações por transtornos mentais e de comportamento decorrentes de substâncias psicoativas: um estudo epidemiológico nacional do período de 1988 a 1999. J Bras Psiquiatr 2002; 5(2): 113-21.

4. Cardinal N. A mulher e a toxicomania. In: Bergeret J, Leblanc J. Toxicomanias: um enfoque pluridimensional. Porto Alegre (RS): Artes Médicas; 1991.

5. Alves Filho N, Alves Júnior JMS, Trindade Filho O. Neonatologia: fisiopatologia e tratamento do recém-nascido. $4^{\mathrm{a}}$ ed. Rio de Janeiro (RJ): Medsi; 1999.

6. Kesmodel U, Wisborg K, Olsen SF, Henriksen TB, Secher NJ. Moderate alcohol intake in pregnancy and the risk of spontaneous abortion. Alcohol Alcohol 2002; 37(1): 87-92. alcoólicas além da permissividade da sociedade. Além disso, a pesquisa permitiu identificar os aspectos psicológicos das gestantes no momento da ingestão de álcool, como sentimentos de felicidade e descontração. Com isso, ficou demonstrado que as mulheres entrevistadas não apresentam um perfil de uso crônico de bebidas alcoólicas, na medida em que a depressão é o diagnóstico mais facilmente encontrado nessas situações.

Também evidenciou-se que apenas metade destas mulheres consumidoras em quantidade moderada disse conhecer os possíveis efeitos da bebida alcoólica para o feto, demonstrando a vinculação inadequada de informações sobre o uso e abuso de substâncias lícitas na gestação, assunto este pouco abordado nas instituições de saúde, nos centros formadores de profissionais e no meio social em geral.

Destaco a importância da assistência de enfermagem no pré-natal com qualidade, na medida em que se constitui em um momento de educação em saúde num amplo espectro, como o de orientar e promover a conscientização das gestantes sobre as possíveis implicações de hábitos não recomendáveis na gestação. Este deve ser aproveitado para implementação de programas educativos, como discussões em grupo, dramatizações de situações cotidianas, relato de experiências, entre outros, com o fim maior de promover a saúde das gestantes por meio da educação e aconselhamento.

Além disso, usando-se como ferramenta a educação em saúde, podem-se evitar não só riscos de complicações obstétricas ou neuromorfológicas sobre o feto de mães consumidoras de bebidas, mas também transtornos emocionais futuros de familiares e, principalmente, da gestante, como a ansiedade vivenciada ao gerar e criar um filho com problemas especiais.

Neste enfoque, a relevância da consulta de enfermagem no pré-natal baseia-se, dentre outras perspectivas, na promoção da saúde da família composta por mulheres em idade reprodutiva. Concordo que a abordagem da assistência de enfermagem deve estar centrada na família, incluindo a avaliação das necessidades de saúde dos membros, a identificação de deficiências e potencialidades de saúde e a intervenção pela educação e aconselhamento com o propósito de incrementar a saúde da familia ${ }^{19}$.

7. Souza GT, Rodrigues MC, Ciavaglia MC. Análise do grau de conhecimento da população sobre a teratogenia do álcool e a conduta de enfermagem. Rev Bras Enferm 1996 abr/jun; 49(2): 287-304.

8. Fabbri CE. Desenvolvimento e validação de um instrumento de rastreamento do uso nocivo de álcool durante a gravidez (T-ACE). [dissertação de mestrado em medicina social] Ribeirão Preto (SP): Faculdade de Medicina de Ribeirão Preto/ USP; 2001.

9. Kaup ZOL, Merighi MAB, Tsunechiro MA. Avaliação do consumo de bebida alcoólica durante a gravidez. Rev Bras Ginecol Obstet 2001 out; 23(9): 575-80.

10. Sokol RJ, Martier SS, Ager JW. The T-ACE questions: practical prenatal detection of risk-drinking. Am J Obstet Gynecol 1989; 160: 863-71.

11. Nóbrega MPSS, Oliveira EM. Dando voz às mulheres usuárias de álcool. Acta Paul Enferm 2003 jul/set; 16(3): 71-80.

12. Freire TM, Machado JC, Melo EV, Melo DG. Efeitos do consumo de bebida alcoólica sobre o feto. Rev Bras Ginecol Obstet 2005; 27(7): 376-81.

13. Neme B. Obstetrícia básica. São Paulo (SP): Sarvier; 1994. 
14. lasi AMK. Ação das drogas no feto e no RN. In: Segre CAM, Armellini PA, Mareno WT. RN. $4^{\text {a }}$ ed. São Paulo (SP): Sarvier; 1995.

15.Chaves Netto H, Sá RAM. Dependência química. In: Chaves Netto, H. Obstetrícia básica. São Paulo (SP): Atheneu; 2004.

16. Zilberman ML. Uso de drogas entre mulheres. In: Baptista M, Cruz MS, Matias R. Drogas e pós-modernidade. Rio de Janeiro(RJ): EDURJ; 2003. vI
17. Belfort P. Medicina preventiva:assistência pré-natal. In: Rezende J. Obstetrícia. $8^{a}$ ed. Rio de Janeiro (RJ): Guanabara Koogan; 1998.

18. Bertolote JM. Problemas sociais relacionados ao consumo deálcool. In: Ramos SP, Bertolote JM. Alcoolismo hoje. $3^{\text {a }}$ ed. Porto Alegre (RS): Artes Médicas; 1997.

19. Branden PS. Enfermagem materno-infantil. $2^{a}$ ed. Rio de Janeiro(RJ): Reichmann \&Affonso; 2000.

\section{ANEXO 1}

\section{Teste de Rastreamento de Consumo de Álcool Durante a Gravidez}

\section{Rastreamento TESTE T-ACE}

\section{T Tolerance: How many drinks does it take to make you feel high?}

Qual a quantidade de bebida alcoólica que você precisa beber para se sentir "alegre" ou descontraída?
( ) 1 dose
( ) 2 doses
( ) 3 doses ou mais

A Have people Annoyed you by criticizing your drinking?

Nos momentos em que você está consumindo bebidas, alguém lhe chama a atenção por você estar "bebendo um pouco a mais?".
( ) $\operatorname{Sim}$
( ) Não

\section{Have you ever felt you ought to Cut down on your drinking?}

Você acha que deve diminuir a quantidade de consumo de bebida alcoólica?

( ) $\operatorname{Sim}($ ) Não

E Eye opener: Have you ever had a drink first thing in the morning to steady your nerves or get rid of a hangover? Ao acordar pela manhã, você costuma beber algo para se sentir bem ou para melhorar o mal-estar por ter consumido bebida alcoólica em excesso?

( ) Sim ( ) Não

PONTOS OBTIDOS NO TESTE T-ACE: 